\title{
Video Article \\ Production and Characterization of Vacuum Deposited Organic Light Emitting Diodes
}

\author{
Daniel de Sa Pereira ${ }^{1}$, Andrew P. Monkman ${ }^{1}$, Przemyslaw Data ${ }^{1,2,3}$ \\ ${ }^{1}$ Physics Department, Durham University \\ ${ }^{2}$ Faculty of Chemistry, Silesian University of Technology \\ ${ }^{3}$ Centre of Polymer and Carbon Materials, Polish Academy of Science
}

Correspondence to: Przemyslaw Data at przemyslaw.data@durham.ac.uk

URL: https://www.jove.com/video/56593

DOI: doi:10.3791/56593

Keywords: Engineering, Issue 141, Thermally Activated Delayed Fluorescence, Organic Electronics, OLED, exciplex, thermal evaporation, ambipolar, Donor-Acceptor, patterning

Date Published: $11 / 16 / 2018$

Citation: de Sa Pereira, D., Monkman, A.P., Data, P. Production and Characterization of Vacuum Deposited Organic Light Emitting Diodes. J. Vis. Exp. (141), e56593, doi:10.3791/56593 (2018).

\section{Abstract}

A method for producing simple and efficient thermally-activated delayed fluorescence organic light-emitting diodes (OLEDs) based on guesthost or exciplex donor-acceptor emitters is presented. With a step-by-step procedure, readers will be able to repeat and produce OLED devices based on simple organic emitters. A patterning procedure allowing the creation of personalized indium tin oxide (ITO) shape is shown. This is followed by the evaporation of all layers, encapsulation and characterization of each individual device. The end goal is to present a procedure that will give the opportunity to repeat the information presented in cited publication but also using different compounds and structures in order to prepare efficient OLEDs.

\section{Video Link}

The video component of this article can be found at https://www.jove.com/video/56593/

\section{Introduction}

Organic electronics brings together all fields from chemistry to physics, going through materials science and engineering in order to improve the current technologies towards more efficient and more stable structures and devices. From this, organic light-emitting diodes (OLEDs) is a technology that has shown great improvements over the last few years, both in terms of efficiency and stability ${ }^{1,2}$. Reports say that the OLED industry for displays may increase from the 16 billion dollars in 2016 to around 40 billion dollars by 2020 and more than 50 billion by $2026^{3}$. It is also finding its way into general lighting and head-mounted microdisplays for augmented-reality ${ }^{4}$. Applications like organic sensors for biomedical applications is more of a futuristic application at the moment, given the requirements for both high luminance and stability ${ }^{5}$. This trend confirms the need for improved device structures that includes more efficient molecules at less expense of natural resources. A better understanding of the inherent processes of the materials used for OLEDs is also of great importance when designing these.

An OLED is a multi-layered organic stack sandwiched between two electrodes, at least one of the latter transparent. Each layer, designed accordingly to their highest occupied molecular orbital (HOMO) and lowest unoccupied molecular orbital (LUMO) and their intrinsic mobility, has a specific function (injection, blockage, and transport) in the overall device. The mechanism is based on opposite charge carriers (electrons and holes) travelling across the device where they meet in a specific layer, recombine to form excitons and from the deactivation of these excitons comes the emission of a photon ${ }^{6}$. This photon will be a characteristic of the layer where the deactivation is taking place ${ }^{7,8,9}$. So, pending molecular design strategies, different red, green and blue emitters can be synthesized and applied to the stack. Putting them together, white devices can also be produced ${ }^{10,11}$. The emitting layer of an OLED stack is usually based on the guest-host $(\mathrm{G}-\mathrm{H})$ system where the guest is dispersed into the host to avoid quenching of light ${ }^{9}$ and by-side reactions ${ }^{12}$.

There are several ways to push molecules to emit light, with thermally-activated delayed fluorescence (TADF) implemented more recently ${ }^{13,14,15}$. TADF allowed for the increase of the external efficiency of devices from $5 \%$ of a typical fluorescence emitter up to $30 \%$ by means of triplet harvesting through a small singlet-triplet energy-splitting in a process called reverse intersystem crossing (rISC). There are several ways to form efficient TADF-based OLEDs: one of the most common in literature is the G-H system where the emissive state is formed by a single molecule ${ }^{16,17,18}$. A second system uses an exciplex emitter formed between an electron donor (D) and an electron acceptor (A) molecules, which are simply called the donor-acceptor (D-A) system ${ }^{15,19,20,21}$; A small range of TADF materials and devices have been reported, yielding very high external quantum yields ${ }^{14}$, reaching a values of, for example, $19 \% \mathrm{EQE}^{22}$, clearly indicating that very efficient triplet harvesting is occurring and that $100 \%$ internal quantum efficiency is possible. In these TADF-based OLEDs, care must be taken when choosing the proper host material as the polarity of the environment can change the charge transfer (CT) state away from the local excited (LE) state, therefore, reducing the TADF mechanism. The procedure to be taken into account is similar to other fluorescent emitters ${ }^{23}$. Such devices have relatively simple stack 
structures, typically 3 to 5 organic layers, and without the need of a $p$-i-n structure ${ }^{24}$, resulting in ultra-low turn-on voltages of the order of $2.7 \mathrm{~V}$ and a maximum thickness of around $130 \mathrm{~nm}$ for all organic layers to guarantee a good charge balance.

Apart from the materials' properties, the production of multi-layered stacks can be either be based on vacuum thermal evaporation (VTE) or spin-coating, the former more frequent for small molecules. It requires precise control over the temperature, pressure, environment, rate, and thickness of each layer. For emitting G-H layers, the rates of co-evaporation have to be controlled for the desired ratios to be obtained. Also of extreme importance is the cleaning of the substrates used for OLEDs which can result in non-working devices or uneven emissions throughout the emitting pixel ${ }^{25}$.

Therefore, this article aims at all steps of preparation, production, and characterization of organic devices and intends to help new specialists on the careful protocol required for high efficiency and evenness of emission. It involves the use of DPTZ-DBTO 2 (2,8-Bis(10H-phenothiazin-10yl)dibenzothiophene-S,S-dioxide) as emitting guest in a TADF G-H system ${ }^{16,26}$. Similar methods can be also implemented for the formation of an exciplex based D-A systems using DtBuCz-DBTO 2 (2,8-Bis(3,6-di-tert-butyl-9H-carbazol-9-yl)dibenzothiophene-S,S-dioxide) in TAPC $\left(4,4^{\prime} \text {-Cyclohexylidenebis[N,N-bis(4-methylphenyl)benzenamine] }\right)^{15}$, where the main difference in the procedure is the concentration ratio of the emissive layer but it significantly changes the nature of emission (single molecule CT emission vs exciplex CT emission). The G-H system described here has a single molecule CT emitter and involves the evaporation of 5 layers with 3 organic, and 2 inorganic materials. The device is composed of indium tin oxide (ITO) as the anode, $40 \mathrm{~nm}$ of $N, N^{\prime}$-di(1-naphthyl)- $N, N^{\prime}$-diphenyl-(1,1'-biphenyl)-4,4'-diamine (NPB) as the hole transport layer $(\mathrm{HTL})$, and a total $20 \mathrm{~nm}$ of $4,4^{\prime}$-bis $\left(N\right.$-carbazolyl)-1,1'-biphenyl (CBP) with $10 \%$ of DPTZ-DBTO ${ }_{2}$ as the emitting layer based on the G-H system. $60 \mathrm{~nm}$ of 2,2',2"-(1,3,5-benzinetriyl)-tris(1-phenyl-1H-benzimidazole) (TPBi) is then used as the electron transport layer $(E T L)$ and $1 \mathrm{~nm}$ of Lithium Floride (LiF) as electron injection layer (EIL). $100 \mathrm{~nm}$ of aluminum (Al) finalizes the device as a cathode. A diagram of the whole procedure can be found in Figure 1. The thicknesses of organics were chosen to be similar to other devices used in the literature. The mobility of each layer must be carefully examined as to ensure good carrier balance inside the layer. The operation of LiF is based on a tunnelling effect, i.e., carriers travel through the tunnels of a packed LiF, ensuring a better injection to the transport layers. This means thin layers (between 0.8 and $1.5 \mathrm{~nm}$ ) are required ${ }^{27}$. The layer of $\mathrm{Al}$ must be thick enough to prevent any oxidation (70 nm is a minimal requirement).

\section{Protocol}

CAUTION: The following procedure involves the use of different solvents, so proper care must be taken when using them. Please use fume and personal protective equipment (gloves, lab-coat). To ensure the quality of the devices evaporated, it is recommended that all the procedure is done in a clean environment (such as a clean room and/or a glovebox). The safety datasheets must be consulted before the use of each equipment/material.

\section{ITO Patterning}

1. Cover uniformly the Indium Tin Oxide $\left(24 \times 24 \mathrm{~mm}^{2}\right.$, ITO-coated glass substrates with a sheet resistance of $20 \Omega / \mathrm{cm}^{2}$ and ITO thickness of $100 \mathrm{~nm}$ ) substrate with a p-type photoresist using a pipette. Spin-coat it at rate $500 \mathrm{rpm}$ for $5 \mathrm{~s}$ followed by $4000 \mathrm{rpm}$ for $45 \mathrm{~s}$.

2. Anneal the substrates on a hotplate for at least $5 \mathrm{~min}$ at $95{ }^{\circ} \mathrm{C}$. This will ensure that all the remaining solvent evaporates producing a uniform film.

3. Place the mask with $4 \mathrm{~mm}$ stripes (or the desired pattern) on the resist-coated ITO substrate. Expose to an $8 \mathrm{~W} 365 \mathrm{~nm}$ UV lamp for $50 \mathrm{~s}$.

4. Place the ITO substrate into the developer solution (1 part developer: 2 parts deionized (DI) water) for $60 \mathrm{~s}$.

5. Carefully rinse the substrate for about $10 \mathrm{~s}$ with a wash bottle containing DI water, holding the substrate with tweezers. Dry the remaining water with an air gun.

6. Heat the ITO substrate on the hotplate at $95^{\circ} \mathrm{C}$ for, at least, $15 \mathrm{~min}$.

7. Remove the photoresist from the edges of the substrate and in-between the stripes using a cotton swab soaked with acetone.

8. Remove the ITO using a mixture of hydrochloric acid and nitric acid $(20: 1 \mathrm{v} / \mathrm{v})$, leave it for $5 \mathrm{~min}$ at room temperature.

9. Rinse with $\mathrm{DI}$ water for $10 \mathrm{~s}$ and remove the rest of photoresist with acetone.

\section{Substrate Cleaning}

1. Take two patterned ITO substrates, rinse for about $10 \mathrm{~s}$ with acetone and wipe with a non-porous sheet of paper or dry with a Nitrogen gun.

2. Using tweezers, fully submerge the substrates into a container with acetone. Put it into an ultrasonic bath ( $320 \mathrm{~W}, 37 \mathrm{kHz})$ for $15 \mathrm{~min}$.

3. Now submerge the substrates into a container with isopropyl alcohol (IPA, 2-propanol). Put the container in the ultrasonic bath for another 15 $\min$.

4. Remove the container from the ultrasonic bath and then the substrates from the IPA bath and dry with a nitrogen gun. Visually inspect the substrates to see if there are no solid residues or smudges. If there is, repeat from point 2.1.

5. Open the flow in the oxygen tank to a rate of around 50 units. Use an oxygen plasma cleaner (100 W, $40 \mathrm{kHz})$ to clean the ITO substrates for 6 min at a $2.5 \mathrm{~L} / \mathrm{h}$ oxygen flow being sure the ITO faces upward.

6. Remove the substrates from the plasma chamber and attach them to the substrate holder. Two masks will be used: (A) for the evaporation of all organic layers and (B) for the evaporation of aluminum (Figure 1). For simplicity, in this protocol, Mask $A$ is attached to the substrate holder

\section{Preparation of the Evaporation Chamber}

1. Insert the substrate holder, mask $A$ and mask $B$, into the evaporation chamber. Depending on the type of the evaporating system, place a substrate holder with mask $A$ on the deposition shelf and mask $B$ on shelf 1. 
2. Add the organic powder of all different materials used for this device in different aluminum oxide crucibles making sure the surface is covered by it. In this case, add NPB, DPTZ-DBTO ${ }_{2}, \mathrm{CBP}$ and TPBi to 4 different $10 \mathrm{~mL}$ aluminum oxide crucibles. Add LiF in one $5 \mathrm{~mL} \mathrm{crucible}$ and aluminum (Al) pieces in a half-full $5 \mathrm{~mL}$ boron nitride high-temperature crucible.

3. Take into consideration the organic crucible position with respective Quartz Crystal Microbalance (QCM) sensor which will give the real value of thickness. For D-A and G-H system evaporations, a co-evaporation process needs to be done. Therefore, to control the co-evaporation process, both DPTZ-DBTO 2 and CBP (TADF OLED) or DtBuCz-DBTO 2 and TAPC (Exciplex OLED) need to be controlled by different QCMs. In this case, the positions of the respective compounds are presented in Figure 2.

4. Close the chamber and initiate the vacuum procedure (also known as pumping down). Wait for the pressure $P<1 \cdot 10^{-5} \mathrm{mbar}$ to start the evaporation.

\section{Evaporation of the Organic Layers}

NOTE: For all organics, do not exceed the evaporation rate of $2 \AA / s$ as this results in increased roughness and decreased uniformity of the layers. To a certain point, this may result in non-uniform emissions and even shorts.

1. Turn $\mathrm{ON}$ the water flow to provide sufficient cooling of the elements.

2. Turn ON the substrate rotation, at 10 rotations per $\min (\mathrm{rpm})$, to ensure the deposition of uniform layers.

3. Pre-heat the NPB crucible by switching-on the temperature controller of the system and open its' shutter. This can be done using the VTE software at the disposal of the user. Start the evaporation (open deposit shutter) when the rate stabilizes at around $1 \AA / \mathrm{s}$. Evaporate a $40 \mathrm{~nm}$ thick layer, close the shutter, wait until the crucible cools down to start the next process.

4. In a similar fashion to point 4.3., preheat the CBP and DPTZ-DBTO ${ }_{2}$ and open their shutters for co-evaporation. Depending on the final concentration of the layer, use different rates of compounds.

1. For the $10 \%$ emissive layer start the evaporation when the rate will stabilize around $2.0 \AA / \mathrm{s}$ for CBP and $0.22 \AA / s$ for $\mathrm{DPTZ}-\mathrm{DBTO}$. Open the depositing shutter when the rate is achieved.

2. Evaporate $20 \mathrm{~nm}$ thickness layer containing $18 \mathrm{~nm}$ of CBP and $2 \mathrm{~nm}$ of DPTZ-DBTO ${ }_{2}$, close the shutter, wait until the crucible cools down to start the next process.

5. Pre-heat the TPBi and open its shutter. Start the evaporation (open deposit shutter) when the rate stabilizes at around $1 \AA /$ s. Evaporate 60 $\mathrm{nm}$ thickness layer, close the shutter, wait until the crucible cools down to start the next process.

6. Pre-heat the LiF, start the evaporation (open evaporation shutter) when the rate stabilizes at around $0.2 \AA /$ s. Do not exceed the evaporation rate of $0.5 \AA / s$. Evaporate $1 \mathrm{~nm}$ thickness layer, close the shutter, wait until the crucible cools down to start next process.

7. Turn OFF the substrate rotation.

8. Replace the mask $A$ on a substrate holder with mask $B$. If necessary, vent the evaporation chamber. If vented, the chamber must be pumped down before the continuation of the procedure. In this protocol, mask $A$ is placed onto mask $B$.

9. Turn ON the substrate rotation, i.e., $10 \mathrm{rpm}$.

10. Pre-heat the Al, start the evaporation (open deposit shutter) when the rate stabilizes at around $1 \AA$ /s. Do not exceed the evaporation rate of 2 $\AA / \mathrm{s}$. Evaporate $100 \mathrm{~nm}$ thickness layer, close the shutter, wait until the crucible cools down.

11. Vent and open the chamber. Remove the substrate holder with deposited devices.

NOTE: Once evaporated, 4 pixels are obtained with two different sizes as shown in Figure 1: $2 \times 4$ and $4 \times 4 \mathrm{~cm}^{2}$. This ensures there is reproducibility when upscaling the devices. The level of defects can also be more visible in the bigger pixels ${ }^{11}$.

\section{OLED Encapsulation}

NOTE: This section is not mandatory for the analysis of OLEDs though it is highly recommended. To secure their quality, it is also important that this section is done in a controlled environment.

1. Remove the substrates from the substrate holder. Place them on top of an encapsulation stage with the evaporated films facing forward.

2. Prepare the resin tube and dispersing tools. Screw a proper tip to one side of the tube, and a pressure gun to the other side of the tube.

3. Apply pressure with the gun to disperse the resin. Draw squares that involve all evaporated pixels (Figure 2).

4. Place an encapsulation glass on top of each square of resin.

5. UV-cure the substrates with the resin and encapsulation glass for as long as required by the resin manufacturer.

\section{OLED Characterization}

1. If necessary, clean the ITO stripes not covered by the encapsulation glass with a toothpick using acetone or IPA to remove any organic material deposited before connecting to the measuring unit. This will guarantee that a good ohmic contact between measuring system and electrodes is achieved.

2. Calibrate the OLED measurement according to the NIST standards using a pre-calibrated lamp.

3. Place the OLED in the integrating sphere, making sure the contacts are placed correctly (Figure 1). Confirm that the anode (+) and cathode $(-)$ are connected to the ITO and Al pads, respectively. Close to the integrating sphere.

4. Measure the I-V curve of the device and the obtained luminance and emission spectra at different voltages.

1. Apply a voltage between the two terminals and measure the current output. A luminance meter measures the brightness output.

2. Using a software and the correct pixel size, calculate the Current Density (J), External Quantum Efficiency (EQE), Power, Wallplug efficiency, Luminous Flux, Luminous efficiency $\left(\eta_{P}\right)$, Current Efficiency $\left(\eta_{L}\right)$ Luminance $(L)$ and Commission Internationale de L'Eclairage (CIE) coordinates. More information on these values can be found in reference ${ }^{15}$.

5. Plot J-V-L, EQE-J, $\eta_{\mathrm{P}}-\mathrm{V}-\eta_{\mathrm{L}}$, EL- $\lambda$ at different voltages and analyse the data. This can be done using a data processing software. For a better understanding, use the following table as a reference on the plotting. 


\section{Representative Results}

The data presented in Figure $\mathbf{3}$ is a good example of the different information one can get by the analysis of this type of OLEDs. From Figure 3a, the turn-on voltage (voltage at which the detector starts detecting light on the device) can be determined. In this case, it is $4 \mathrm{~V}$. Device degradation due to high voltages is seen when luminance decreases substantially (around $13 \mathrm{~V}$ ). Degradation occurs when carriers injected into the device react with the organic layers resulting in the breaking of bonds and molecules. Also, electrical stress can be associated with device degradation. The maximum luminance of this device is around $17000 \mathrm{~cd} / \mathrm{m}^{2}$. From Figure $3 \mathbf{b}$, the maximum E.Q.E. (around $7 \%$ ) and roll-off, a measure of the device electrical stability, are determined. The roll-off of a device is also defined as the drop in efficiency with the current flowing through it. To compare the roll-off of different devices, the values of EQE at the standard luminance of 100 and $1000 \mathrm{~cd} / \mathrm{m}^{2}$ are usually given ${ }^{6}$. In this case, 6.1 and $5.5 \%$, respectively which represents a drop of $9 \%$ and $20 \%$ of its maximum value. This represents a poor roll-off. Good values should be between 0 and $5 \%$ until high levels of luminance. The other values of efficiency are shown in Figure 3c, as other means of comparison with similar types of devices. Finally, the EL is shown peaking at $573 \mathrm{~nm}$, a typical green-yellow emission (inset of Figure $3 d$ ). The EL at different voltages can help giving insights into optical stability i.e., where the emission is taking place. In this case, as this seemingly does not change with the applied voltage, one can assume that the device is optically-stable. Checking the CIE coordinates (inset of Figure $\mathbf{3 b}$ with voltage is another way to measure the optical stability.

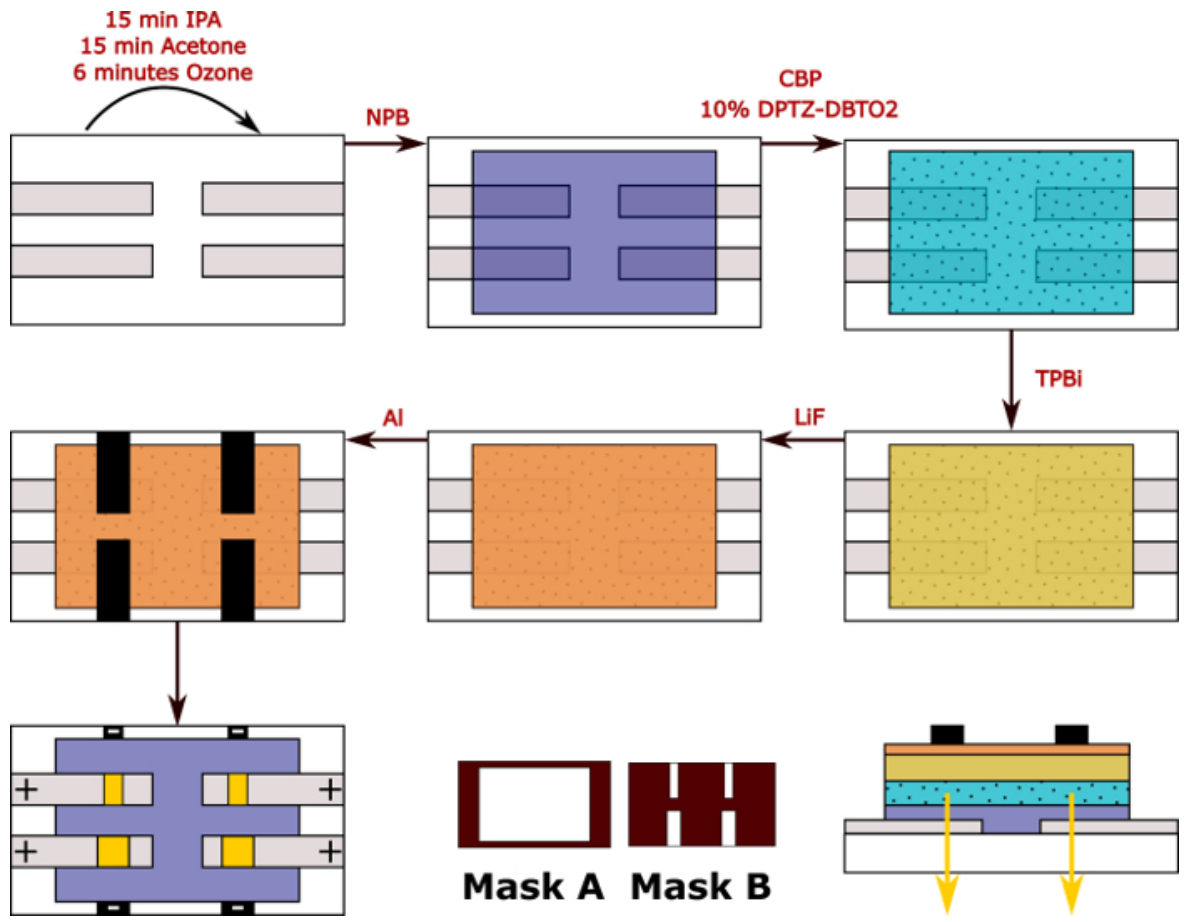

Figure 1: Diagram containing all steps represented in this protocol. All organic layers and LiF are evaporated using mask A. After metallization (evaporation of aluminium), two sets of devices can be produced using mask B: one with $2 \times 4 \mathrm{~cm}^{2}$ and another with $4 \times 4 \mathrm{~cm}^{2}$. The voltage will be applied between the ITO (anode: +) and aluminium (cathode: - ) and a current will be measured. A cross-section of the device structure is also shown. Please click here to view a larger version of this figure.
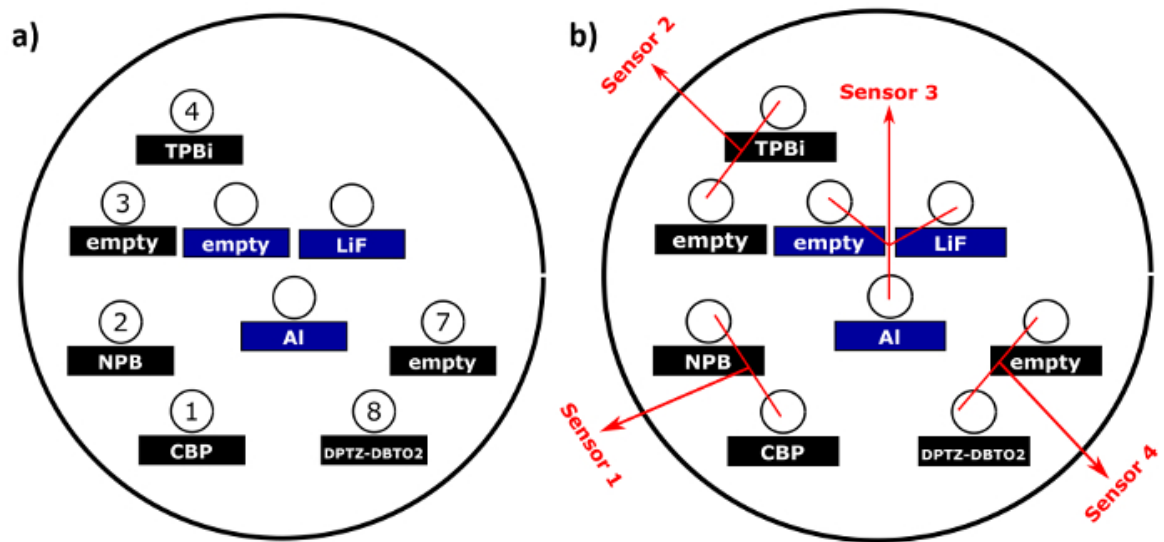

Figure 2: a) Diagram of the organic low temperature (black) and inorganic high-temperature sources (blue) to be placed in the vacuum chamber. Each material has to be put in the specified source with a specific heating number for the software as they were previously optimized for each material in question. b) QCM sensors arranged throughout the chamber. Please click here to view a larger version of this figure. 

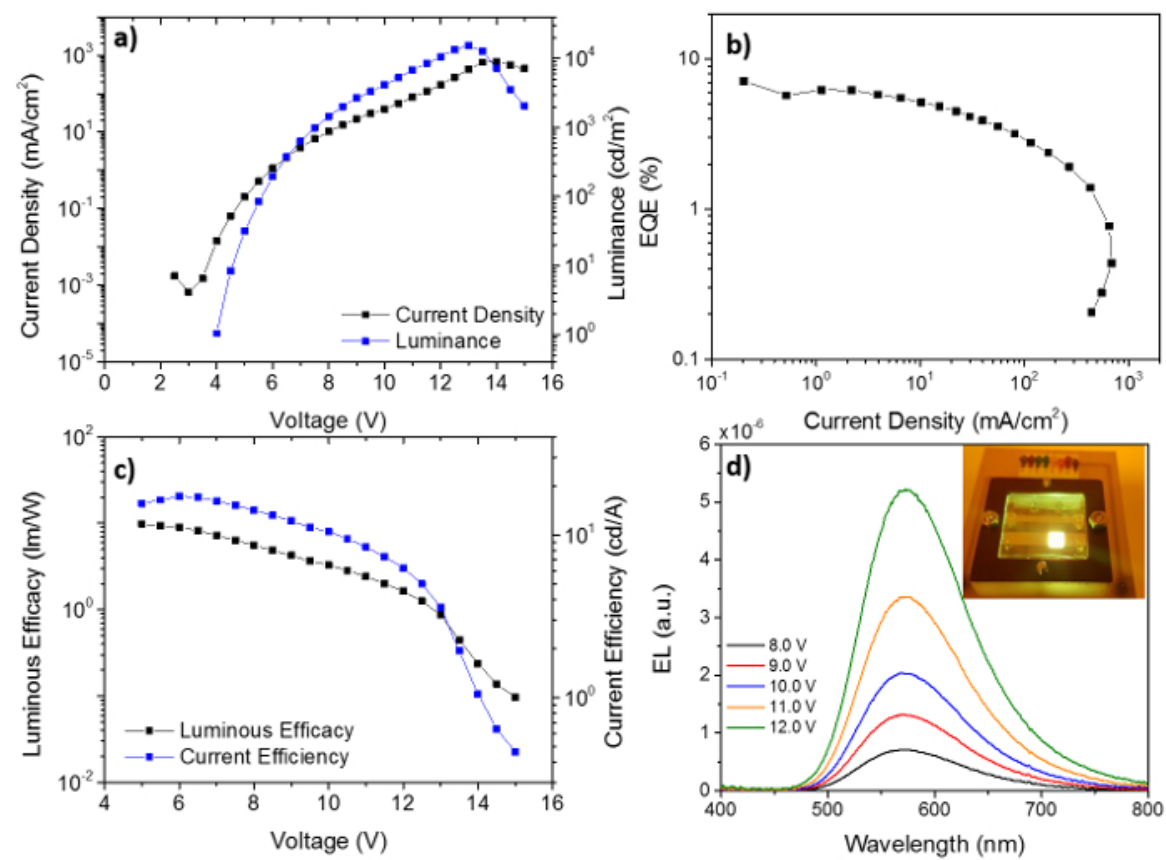

Figure 3: a) J-V-L, b) EQE-J, c) $\eta P-V-\eta L$, d) EL- $\lambda$ at different voltages for the device in this study. The CIE coordinates change with voltage is shown on the inset of $\mathbf{b}$ ) while a photograph of the device is shown in the inset of $\mathbf{d}$ ). Please click here to view a larger version of this figure.

\begin{tabular}{|c|c|c|c|c|c|c|}
\hline Curve & $x$ & Scale & $\mathrm{y} 1$ & Scale & y2 & Scale \\
\hline J-V-L & \multirow[t]{2}{*}{$\mathrm{V}$} & \multirow[t]{2}{*}{ linear } & $\mathrm{J}$ & $\log$ & $L$ & $\log$ \\
\hline$\eta_{P}-V-\eta_{L}$ & & & $\eta_{P}$ & $\log$ & $\eta_{L}$ & $\log$ \\
\hline EQE-J & $\mathrm{J}$ & $\log$ & EQE & $\log$ & & \\
\hline EL- $\lambda$ & $\lambda$ & linear & $\mathrm{EL}$ & linear & & \\
\hline
\end{tabular}

Table 1: Considered curves and related scale for the unification of the characterization of OLEDs.

\section{Discussion}

The present protocol aims to present an effective tool for the patterning, production, encapsulation and characterization of OLEDs based on small molecular-weight TADF-emitting or exciplex-emitting layers. The organic vacuum thermal evaporation allows for the production of thin films (from a few $\AA$ to hundreds of $\mathrm{nm}$ ) of both organic and inorganic materials and produce pathways for charge carriers to recombine from which light will be emitted. Although versatile, the device production is fairly limited to the evaporator i.e., the number of organic and inorganic sources available or the possibility of more than one evaporation at the same time (co- and tri-evaporations are very common, particularly in TADF devices). More advanced systems may allow for the evaporation of more than 3 sources at the same time, which may be useful for applications such as white-OLEDs ${ }^{28}$ for displays and general lighting. Nevertheless, a trade-off between the device complexity and its performance must be met. The multifunctionality of this evaporation procedure also allows doing different studies that go beyond this work. These include effects of layer thickness, dopant concentration, layer functionality or even study the inherent mobilities of new layers. The fine control over the rates of single and co-evaporated layers is also crucial since it allows for the formation of uniform films with controlled precise rations.

It is recommended that all steps of this protocol are done in a controlled environment and, more importantly for the encapsulation, inside a glovebox to avoid any ambient related degradation. Finally, an integrating sphere is most welcomed as it provides for a more detailed electrical and optical analysis. With this mind, all steps from theoretical introduction to production and characterization of TADF-based OLEDs were presented in this protocol highlighting all these different stages allowing the production of stable devices that, when encapsulated, can last for large periods of time.

\section{Disclosures}

The authors have nothing to disclose.

\section{Acknowledgements}

The authors would like to acknowledge the "Excilight project" which received funding from H2020-MSCA-ITN-2015/674990. 


\section{References}

1. Tang, C. W., \& VanSlyke, S. A. Organic electroluminescent diodes. Applied Physics Letters. 51, 913-915 (1987).

2. Shin, H. et al. Sky-Blue Phosphorescent OLEDs with $34.1 \%$ External Quantum Efficiency Using a Low Refractive Index Electron Transporting Layer. Advanced Materials. 1-6 (2016).

3. Tremblay, J.-F. The rise of OLED displays. C\&EN. Available at: <http://cen.acs.org/articles/94/i28/rise-OLED-displays.html> (2016)

4. Bardsley, N. et al. Solid-State Lighting R\&D Plan. (2016).

5. Richter, B., Vogel, U., Herold, R., Fehse, K., Brenner, S., Kroker, L., Baumgarten, J. Bidirectional OLED Microdisplay: Combining Display and Image Sensor Functionality into a Monolithic CMOS chip. in IEEE. 314 (2011).

6. Sa Pereira, D., Data, P., \& Monkman, A. P. Methods of Analysis of Organic Light. Display and Imaging. 2, $323-337$ (2017).

7. Lin, T.-A. et al. Sky-Blue Organic Light Emitting Diode with $37 \%$ External Quantum Efficiency Using Thermally Activated Delayed Fluorescence from Spiroacridine-Triazine Hybrid. Advanced Materials. (2016).

8. Dos Santos, P. L., Ward, J. S., Bryce, M. R., \& Monkman, A. P. Using Guest-Host Interactions to Optimize the Efficiency of TADF OLEDs. Journal of Physical Chemistry Letters. 7, 3341-3346 (2016).

9. Jou, J.-H., Kumar, S., Agrawal, A., Li, T.-H., \& Sahoo, S. Approaches for fabricating high efficiency organic light emitting diodes. Journal of Materials Chemistry. C. 3, 2974-3002 (2015).

10. de Sa Pereira, D. et al. An optical and electrical study of full thermally activated delayed fluorescent white organic light-emitting diodes. Scientific Reports. 7, (2017).

11. Pereira, D., Pinto, A., California, A., Gomes, J., \& Pereira, L. Control of a White Organic Light Emitting Diode's emission parameters using a single doped RGB active layer. Materials Science and Engineering: B. 211, 156-165 (2016).

12. Data, P. et al. Evidence for Solid State Electrochemical Degradation Within a Small Molecule OLED. Electrochimica Acta. 184, 86-93 (2015).

13. Uoyama, H., Goushi, K., Shizu, K., Nomura, H., \& Adachi, C. Highly efficient organic light-emitting diodes from delayed fluorescence. Nature. 492, 234-8 (2012).

14. Goushi, K., Yoshida, K., Sato, K., \& Adachi, C. Organic light-emitting diodes employing efficient reverse intersystem crossing for triplet-tosinglet state conversion. Nature Photonics. 6, 253-258 (2012).

15. Jankus, V. et al. Highly efficient TADF OLEDs: How the emitter-host interaction controls both the excited state species and electrical properties of the devices to achieve near 100\% triplet harvesting and high efficiency. Advanced Functional Materials. 24, 6178-6186 (2014).

16. Etherington, M. K. et al. Regio- and conformational isomerization critical to design of efficient thermally-activated delayed fluorescence emitters. Nature Communications. 8, 14987 (2017).

17. Okazaki, M. et al. Thermally activated delayed fluorescent phenothiazine-dibenzo[a,j]phenazine-phenothiazine triads exhibiting tricolorchanging mechanochromic luminescence. Chemical Science. 8, 2677-2686 (2017).

18. Suzuki, Y., Zhang, Q., \& Adachi, C. A solution-processable host material of 1,3-bis\{3-[3-(9-carbazolyl)phenyl]-9-carbazolyl\}benzene and its application in organic light-emitting diodes employing thermally activated delayed fluorescence. Journal of Materials Chemistry. C. 3 , 1700-1706 (2015).

19. Data, P. et al. Efficient p-phenylene based OLEDs with mixed interfacial exciplex emission. Electrochimica Acta. 182, 524-528 (2015).

20. Data, P. et al. Exciplex Enhancement as a Tool to Increase OLED Device Efficiency. Journal of Physical Chemistry C. 120, 2070-2078 (2016).

21. Data, P. et al. Dibenzo[a,j]phenazine-Cored Donor-Acceptor-Donor Compounds as Green-to-Red/NIR Thermally Activated Delayed Fluorescence Organic Light Emitters. Angewandte Chemie International Edition. 55, 5739-5744 (2016).

22. Goushi, K., \& Adachi, C. Efficient organic light-emitting diodes through up-conversion from triplet to singlet excited states of exciplexes Applied Physics Letters. 23306, 10-14 (2014).

23. Dos Santos, P. L. et al. Engineering the singlet-triplet energy splitting in a TADF molecule. Journal of Materials Chemistry. C. 4, 3815-3824 (2016).

24. He, G. et al. Very high-efficiency and low voltage phosphorescent organic light-emitting diodes based on a p-i-n junction. Journal of Applied Physics. 95, 5773-5777 (2004).

25. Pereira, L. in Organic light emitting diodes: the use of rare earth and transition metals. 33-36 Pan Stanford (2011).

26. Dias, F. B. et al. The Role of Local Triplet Excited States in Thermally-Activated Delayed Fluorescence: Photophysics and Devices. Advanced Science. 3, 1600080 (2016).

27. Kim, Y. Power-law-type electron injection through lithium fluoride nanolayers in phosphorescence organic light-emitting devices. Nanotechnology. 19, 0-4 (2008).

28. Reineke, S., Thomschke, M., Lüssem, B., \& Leo, K. White organic light-emitting diodes: Status and perspective. Reviews of Modern Physics. 85, 1245-1293 (2013). 